\title{
Accretion Disks and Dynamos: Toward a Unified Mean Field Theory
}

\author{
Eric G. Blackman ${ }^{1}$ \\ 1. Dept. of Physics and Astronomy, University of Rochester, Rochester NY 14627, USA; \\ blackman@pas.rochester.edu
}

\begin{abstract}
Conversion of gravitational energy into radiation near stars and compact objects in accretion disks and the origin of large scale magnetic fields in astrophysical rotators have often been distinct topics of active research in astrophysics. In semi-analytic work on both problems it has been useful to presume large scale symmetries, which necessarily results in mean field theories; magnetohydrodynamic turbulence makes the underlying systems locally asymmetric and highly nonlinear. Synergy between theory and simulations should aim for the development of practical, semi-analytic mean field models that capture the essential physics and can be used for observational modeling. Mean field dynamo (MFD) theory and alpha-viscosity accretion disc theory have exemplified such ongoing pursuits. Twenty-first century MFD theory has more nonlinear predictive power compared to 20th century MFD theory, whereas alpha-viscosity accretion theory is still in a 20th century state. In fact, insights from MFD theory are applicable to accretion theory and the two are really artificially separated pieces of what should ultimately be a single coupled theory. I discuss pieces of progress that provide clues toward a unified theory. A key concept is that large scale magnetic fields can be sustained via local or global magnetic helicity fluxes or via relaxation of small scale magnetic fluctuations, without appealing to the traditional kinetic helicity driver of 20th century textbooks. These concepts may help explain the formation of large scale fields that supply non-local angular momentum transport via coronae and jets in a unified theory of accretion and dynamos. In diagnosing the role of helicities and helicity fluxes in disk simulations, it is important to study each disk hemisphere separately to avoid being potentially misled by the cancelation that occurs as a result of reflection asymmetry. The fraction of helical field energy in disks is expected to be small compared to the total field in each hemisphere as a result of shear, but can still play a fundamental role in large scale dynamo action.
\end{abstract}

Subject headings: accretion, accretion disks — black hole physics — instabilities — MHD — turbulence 


\section{Introduction}

Large scale dynamo (LSD) theory in astrophysics is aimed at quantitatively understanding the in situ physics of magnetic field growth, saturation, and sustenance on time or spatial scales large compared to turbulent scales of the host. The presence of magnetohydrodynamic turbulence makes the subject particularly rich, highly nonlinear, and exacerbates the importance of numerical simulations. However, large scale spatial symmetry and slow evolution of large scale fields compared to turbulent fluctuations motivates a mean field approach in which statistical, spatial, or temporal averages are taken and evolution of the mean field studied (e.g. Moffatt 1978).

An important goal of semi-analytic mean field dynamo (MFD) theory is to capture the essential nonlinear physics simply enough for phenomenological models, but rich enough to account for the nonlinear saturation found in simulations (for technical reviews see: e.g. Brandenburg \& Subramanian 2005; Blackman 2007). The 21st century has brought significant progress in this endeavor as the growth and saturation of LSDs seen in simple simulations seem to be reasonably matched by newer MFD theories that follow the time dependent dynamical evolution of magnetic helicity (e.g. Blackman \& Field 2002). The modern 21st century MFDs represent two or three scale theories that capture the basic principles of the inverse cascade of magnetic helicity first derived in the spectral model of Pouquet et al. (1976), which revealed the fundamental utility of tracking magnetic helicity evolution to understand large scale field growth.

The existence of LSDs is not controversial: The sun proves that in situ LSDs operate in nature since its large scale field reverses its sign over each half solar cycle (e.g. Wang \& Sheely 2003; Schrijver \& Zwaan 2000). If the field were simply that frozen into to the ISM during initial formation, there would be no reversals. LSDs are also likely operating in galaxies, despite whatever initial fields may have been seeded cosmologically because supernova driven turbulent diffusion would otherwise diffuse the large field. Detailed modeling of the geometry of galactic large scale fields can be matched by mean field theories (Beck et al. 1996; Vallée 2004; Shukurov 2002), though incorporation of the nonlinear LSD principles of the past decade for idealized dynamos into more geometrically realistic dynamos of astrophysical rotators with realistic boundary conditions setting is an ongoing avenue of research (e.g. Sur et al. 2007,2009).

Given the role of LSDs in stratified rotating stars and galaxies, their presence in turbulent accretion discs seems likely. Large scale fields likely play an integral role in the angular momentum transport for many real systems and this is evidenced by the presence of coronae and jets. The global scale fields required by standard models of astrophysical jets (e.g. Blandford \& Znajek 1977; Blandford \& Payne 1982) may be produced by an in situ LSD, although understanding the relative importance of in situ production vs. flux accretion in that context has been a long-standing subject of research (e.g. Lovelace et al. 2009; Beckwith et al. 2011). Non-thermal coronal luminosity from accretion engines also hints at the role of LSDs because only large scale magnetic structures can survive the buoyant rise to the corona without being dissipated in the disk if the latter 
is turbulent (Blackman \& Pessah 2009). Indeed, magnetohydrodynamic turbulence is expected in accretion discs (e.g. Balbus \& Hawley 1998). If all of the large scale fields in the corona and jet were produced in the disk, then understanding the physics of coronal relaxation is an important part of assessing what fraction becomes the global jet fields and what fraction produces smaller loops that dissipate in the corona.

Amplification of large scale fields is now routinely seen in a wide range of accretion-diskmotivated simulations: Localized stratified shearing box simulations indeed show evidence for large scale field reversing LSD cycles (Brandenburg et al. 1995; Lesur \& Ogilive 2008; Davis et al. 2010; Guan \& Gammie 2011). Global simulations, now becoming more computationally feasible, also show such LSD cycles and the large scale magnetic fields either in coronae or jets (e.g. Beckwith et al. 2011, Sorathia et al. 2010, Tchekhovskoy et al. 2011, Romanova et al. 2011) though criteria for convergence (Hawley et al. 2011; Bodo et al. 2011) and sensitivity of the dynamical importance of associated vertical angular momentum transport on the initial conditions (e.g. compare Penna et al. 2010 and Tchekhovskoy et al. 2011). must be further investigated. The stress gradients associated with the large scale fields in real systems may be significant contributors to angular momentum transport that act in symbiosis with with local turbulent transport. As will be discussed later, an important point is that LSDs need not depend on the presence of kinetic helicity as a driver. In fact the electromotive force needed for LSDs can be magnetic fluctuation driven (Blackman \& Field 2004) or even helicity flux driven (Blackman \& Field 2000a, Vishniac \& Cho 2001; Subramanian \& Brandenburg 2005) These concepts are important for magneto-rotational instability (MRI) unstable systems (Balbus 2003) because magnetic fluctuations typically exceed the velocity fluctuations and differential shear can conspire to produce non-negligible helicity fluxes (Vishniac 2009).

While LSD theory has always been presented with explicit mean field theories that expose the approximations made, axisymmetric accretion disc models such as the Shakura Sunyaev (Shakura \& Sunyaev 1973; hereafter SS73) viscous $\alpha_{s s}$ model are not always recognized as mean field theories by those using them to model spectra. In this sense, 21 st century LSD theory is more progressed than axisymmetric mean field accretion disc theory: The $\alpha_{s s}$ disc prescription does not yet offer predictive power for numerical simulations, whereas 21 st century LSD theory predicts the saturation of LSDs seen in a range of simulations. Ironically, because large scale fields likely play a fundamental role in accretion discs, both MFD and accretion theory are really two faces of a single coupled theory.

The subjects of LSD theory and accretion involve the study of intrinsically time dependent non-equilibirum processes and are in direct alignment with the themes of the "Turbulent Mixing and Beyond" 2011 meeting: Turbulent "mixing" in the LSD context takes the form of turbulent diffusion of large scale magnetic fields. Part of the study of LSDs involves understanding how large scale growth mechanisms compete with turbulent diffusion and what role turbulent transport plays 
in establishing large scale field geometries and cycle periods. In accretion disks, turbulent diffusion, or more generally, MHD mediated turbulent transport, has long been thought to be involved in at least some of the necessary extraction of angular momentum. It is likely that the transport of large scale fields and transport of angular momentum are intimately linked in magnetized accretion disks. This collection of integrated topics continues to benefit from symbioses between large-scale numerical simulations, experiments, and basic theory.

Below I first review conceptual progress of 21 st century LSD/MFD theory. I then discuss parallels between LSD theory and accretion disc theory. Finally, I discuss emerging connections between the two how they should be unified into one.

\section{21st Century LSD Theory}

For $\sim 50$ years, 20th century textbook MFD theory (e.g. Moffatt 1978) lacked a saturation theory to predict how strong the large scale fields get before quenching via the back-reaction of the field on the driving flow. But substantial progress toward a nonlinear mean field theory has emerged in the 21 st century via a symbiosis between analytical and numerical work. Coupling the dynamical evolution of magnetic helicity into the dynamo equations turns out to provide fundamental insight to the saturation seen in simulations. Whether this approach is the only way to understand the saturation is uncertain, but it has been fruitful.

The connection between magnetic helicity and large scale dynamos is first evident in the classic EDQNM spectral model of helical MHD turbulence in Pouquet et al. (1976). In Kleeorin \& Ruzmaikin (1982) an equation that couples the time evolution of small scale magnetic helicity to the LSD was given but not solved. A time-dependent two scale LSD model that incorporates the principles of Pouquet et al. (1976) was derived and solved in Blackman \& Field (2002), and Field \& Blackman (2002) showed that solutions matched simulations of Brandenburg (2001). For a

variety of different discussions see Brandenburg \& Subramanian (2005); Blackman (2007); Käpylä et al. 2008) and below.

\subsection{Globally Helical}

Most work in LSD/MFD theory has focused on systems that are initially globally reflection asymmetric (GRA). In such systems, pseudoscalars, such as the hemispherically averaged product of angular velocity and density gradient imposed by the initial setup facilitate e.g. a kinetic helicity pseudoscalar, common to standard textbooks (Moffatt 1978; Parker 1979) " $\alpha_{d y n}$ effect" of mean field dynamos. However, 21st century MFD theory has revealed that magnetic helicity and its evolution provides a more fundamental unifying quantity for all MFDs. The initial GRA conditions and the potential pseudoscalars that arise are important primarily because they provide one way to 
sustain an electromotive force aligned with the mean magnetic field. This alignment is a source of magnetic helicity or magnetic helicity flux.

There are two classes of GRA LSDs. The first is flow dominated helical dynamos (FDHD) which apply inside of astrophysical rotators. Here the initial energy is dominated by velocity flows and thermal energy. The magnetic field may grow larger than the fluctuating velocities on some scales, but the total kinetic energy in all forms remains larger than the total magnetic energy in FDHDs.

The FDHDs are potentially linked to coronae via boundary terms where a second type of LSD, the magnetically driven helical dynamo (MDHD) can operate. A MDHD can also be described as dynamical magnetic relaxation (Blackman \& Field 2004) toward the "Taylor" state (Taylor 1986). Note that the Taylor state is the lowest energy state to which a magnetically dominated configuration evolves subject to boundary conditions. For a closed system, the state is characterized by the magnetic helicity having migrated to the largest scale available. In an accretion disc, the MDHD would characterize coronal relaxation of magnetic structures (fed by helical fields from below) into larger (even jet mediating) scales in a magnetically dominated environment. In this respect, the MDHD in disc-corona or star-corona interfaces are analogous to laboratory plasma dynamos ((Bellan 2000; Ji \& Prager 2002)) that occur in reverse field pinches (RFPs) and Spheromaks. The corona becomes the "internal volume" of these devices and the disc or stellar surface acts as the injecting boundary.

LSDs always involve some helical growth of the large scale field, which is coupled to a helical growth of smaller scale fields of opposite sign and/or a compensating helicity flux when boundary conditions allow. When small scale magnetic or current helicity evolution is coupled to the large scale field growth, the simplest 21st century dynamo reveals that the mean field 'dynamo $\alpha_{d y n}$ becomes the difference between kinetic helicity and current helicity: For an $\alpha_{d y n}^{2}$ type FDHD simulated in a closed box (Brandenburg 2001), the current helicity builds up as the large scale field grows and quenches the FDHD, in accordance with MFD predictions (Blackman \& Field 2002;).

The effect of boundary or flux terms can vary depending on the sign and relative flux of small and large scale magnetic helicities. For a closed system the buildup of small scale magnetic (and current) helicity is necessarily accompanied by small scale magnetic helicity buildup with the opposite sign, which quenches the LSD to at most a rate determined by the dreadfully slow resistive dissipation of small-scale helicity. If this "catastrophic" quenching occurs before enough large scale field is grown in the fast growth regime, a preferential flux of helicity of the opposite sign through a realistic astrophysical boundary is desirable to alleviate this quenching and sustain further fast fast growth. For GRA systems, in each hemisphere, the large scale helicity builds with one sign and the small scale with the opposite sign so an outward flux of small scale helicity is desirable to sustain the electromotive force (Blackman \& Field 2000ab; Vishniac \& Cho 2001; Sur et al. 2007; Käpylä et al 2008). It is possible that even if the field grows to acceptable magnitudes 
without boundary fluxes, the cycle period becomes resistively limited. In this case the helicity fluxes can unclog the cycle. I will come back to the role of helicity fluxes in section 3.2.

For an MDHD, the system is first dominated instead by the current helicity and a growing kinetic helicity can act as the back-reaction (Blackman \& Field 2004; Park \& Blackman 2012). Both simple FDHDs and MDHDs are accessible within the same formalism, all unified by tracking magnetic helicity evolution, and aided by thinking of the field as ribbons rather than lines (Blackman \& Brandenburg 2003).

Interestingly, although most observations probe coronare, most work on astrophysical dynamo theory has focused on the FDHD and not the MDHD.

\subsection{Not Globally Helical}

LSD action has also been observed in non GRA simulations (e.g. Yousef et al 08; Lesur \& Ogilvie 08; Brandenburg et al. 2008). The minimum global ingredients for this class of LSD seem to be shear plus turbulence (Yousef et al. 2008). The non GRA LSDs grow large scale fields on scales larger than the outer scale of turbulence but smaller than the global scale. Although there is no GRA, in regions where the large scale field is coherent regions, there is a field aligned electromotive force (EMF), and thus an intermediate scale source of magnetic helicity that may switch signs between coherence regions and globally averages to zero. The most promising explanation for the Yousef et al. (2008) simulations seems to be that of Heinemann et al. (2011) which involves fluctuations in the electromotive force. This is not unrelated to the implications of a stochastic $\alpha$ effect (e.g. Vishniac \& Brandenburg 1997 [stochastic $\alpha_{d y n}$ effect]). When there are no helical fluctuations in the EMF and no pseudoscalars, shear alone does produce an LSD (Kolekar et al. 2012). I would also point out that in sub-global patches where any mean field grows and the EMF is finite for a given time span, its alignment with the local mean magnetic field means it is a source of large scale magnetic helicity locally. In general, the absence of GRA does not imply the irrelevance of magnetic helicity and/or its flux, in local subregions of a volume for which the global flux may vanish.

\section{Why Tracking Magnetic Helicity in Large Scale Dynamos Provides Insight}

The time evolution of the mean field $\overline{\mathbf{B}}$ is given by the induction equation (Moffatt 1978; (Krause \& Rädler 1980))

$$
\frac{\partial \overline{\mathbf{B}}}{\partial t}=-c \nabla \times \overline{\mathbf{E}},
$$

where

$$
c \overline{\mathbf{E}}=-\overline{\mathbf{V}} \times \overline{\mathbf{B}}-\langle\mathbf{v} \times \mathbf{b}\rangle+\nu_{M} \nabla \times \overline{\mathbf{B}},
$$


where the magnetic diffusivity $\nu_{M} \equiv \frac{\eta c^{2}}{4 \pi}$ and $\eta$ is the resistivity. The turbulent electromotive force $\overline{\mathcal{E}} \equiv\langle\mathbf{v} \times \mathbf{b}\rangle$ can be expanded in terms of powers of spatial and temporal derivates of $\overline{\mathbf{B}}$ and $\overline{\mathbf{V}}$ (or mean shear). The lowest order contributions to $\overline{\mathcal{E}}$ from the mean magnetic field that lead to exponential growth and diffusion (and are the only contributions in the absence of a mean velocity) are given by

$$
\overline{\mathcal{E}}_{i}=\alpha_{i j} \bar{B}_{j}-\beta_{i j k} \partial_{j} \bar{B}_{k} .
$$

Using Maxwell's equations, the definitions $\nabla \times \mathbf{A} \equiv \mathbf{B}$, and $\mathbf{J} \equiv \frac{c}{4 \pi} \nabla \times \mathbf{B}$, along with vector identities, the equation for the evolution of magnetic helicity density $H^{M} \equiv\langle\mathbf{A} \cdot \mathbf{B}\rangle$ is (e.g. Bellan 2000)

$$
\partial_{t}(\mathbf{A} \cdot \mathbf{B})=-2 \nu_{M}(\mathbf{J} \cdot \mathbf{B})-\nabla \cdot\left(2 \Phi \mathbf{B}+\mathbf{A} \times \partial_{t} \mathbf{A}\right),
$$

where $\Phi$ satisfies $\mathbf{E}=\nabla \Phi-\frac{1}{c} \frac{d \mathbf{A}}{d t}$. Following the analogous procedure for the mean and fluctuating quantities, and using (2) and ((3) gives for the large and small scale quantities $H_{1}^{M} \equiv\langle\overline{\mathbf{A}} \cdot \overline{\mathbf{B}}\rangle$ and $H_{2}^{M} \equiv\langle\mathbf{a} \cdot \mathbf{b}\rangle$ respectively

$$
\partial_{t}(\overline{\mathbf{A}} \cdot \overline{\mathbf{B}})=2 \overline{\mathcal{E}} \cdot \overline{\mathbf{B}}-2 \nu_{M} \overline{\mathbf{J}} \cdot \overline{\mathbf{B}}-\nabla \cdot\left(2 \bar{\Phi} \overline{\mathbf{B}}+\overline{\mathbf{A}} \times \partial_{t} \overline{\mathbf{A}}\right)
$$

and

$$
\partial_{t} \overline{\mathbf{a} \cdot \mathbf{b}}=-2 \overline{\mathcal{E}} \cdot \overline{\mathbf{B}}-2 \nu_{M} \overline{\mathbf{j} \cdot \mathbf{b}}-\nabla \cdot\left(\overline{2 \phi \mathbf{b}}+\overline{\mathbf{a} \times \partial_{t} \mathbf{a}}\right) .
$$

Standard MFDs necessarily involve generation of mean magnetic helicity. To see this, note that the minimalist versions of such dynamos invoke $\alpha_{i j}=\alpha_{d y n} \delta_{i j}$ and $\beta_{i j k}=\nu_{M, T} \epsilon_{i j k}$ where $\alpha_{d y n}$ is a pseudoscalar and $\nu_{M, T}$ acts as a mean field scalar diffusion coefficient. The growth of the large scale field then involves a finite $\overline{\mathcal{E}} \cdot \overline{\mathbf{B}}$, which provides an equal and opposite source term to the large scale and small scale magnetic helicity as seen from (5) and (6). In solving (1), standard 20th century textbook theory focuses on an equation for the large scale field from which one can write an equation like (5). But 20th century dynamo theory does not couple in the evolution of the small scale magnetic helicity contained in (6).

In the simplest 21 st century LSDs which consolidates aspects of the spectral study of Pouquet et al. (1976) into two scales (e.g. Field \& Blackman 2002; Blackman \& Field 2002; Blackman \& Brandenburg 2002), $\alpha_{d y n} \sim-\tau(\langle\mathbf{v} \cdot \nabla \times \mathbf{v}\rangle-\langle\mathbf{b} \cdot \nabla \times \mathbf{b}\rangle) \equiv \alpha_{k i n}+\alpha_{m a g}$, where $\tau$ is of order a correlation time of the dominant fluctuations. In 20th century versions of helical dynamos (Moffatt et al. 1978) only $\alpha_{k i n}$ contributes to $\alpha_{d y n}$. The contribution $\alpha_{m a g}$ is proportional to the small scale current helicity, which in turn is proportional to $\mathrm{H}_{2}^{M}$ in the Coulomb gauge for a closed system. Therefore the time evolution of $\alpha_{d y n}$ is directly coupled to the time evolution of magnetic helicity. If $H_{1}^{M}$ grows initially from $\alpha_{k i n}$, then $H_{2}^{M}$ grows of opposite sign which in turn quenches $\alpha_{d y n}$. 


\subsection{Kinetic vs. Magnetic Fluctuation Driven LSDs}

Comparisons between theory and numerical experiments have validated the basic theoretical framework of 21 st century LSD just described. The simplest experiments are those of the so called $\alpha^{2}$ dynamo in which isotropically driven kinetic helicity is injected into a periodic box at intermediate wave number $k \sim 5$, and the $k=1$ large scale field grows (Brandenburg 2001). Agreement between the basic theory and simulations of such kinetically forced (KF) dynamos has been shown in (Field \& Blackman 2002; see also Brandenburg \& Subramanian 2005; Park \& Blackman 2012a). The insight gained from comparison of the basic $\alpha^{2}$ LSD theory to the simulations is that when kinetic helicity is forced and drives large scale helical magnetic fields, the small scale helical field grows because magnetic helicity is largely conserved up to resistive terms. The buildup of the small scale current helicity associated with the small scale field then off-sets the driving kinetic helicity and quenches the LSD. Ultimately the slightly faster resistive decay of the small scale field than the large scale field leads to an asymptotic steady state with net helicity associated with that of the large scale sign.

However, this physical mechanism and the equations that describe it also motivate consideration of the complementary case of driving the initial system with current helicity rather than kinetic helicity. This was investigated analytically in Blackman \& Field (2004) where it was found that driving the system so as to supply initial small scale current helicity produced a magnetically forced (MF) analogue to the $\alpha^{2}$ dynamo. Because the MF case is driven by magnetic fluctuations, there is no "kinematic" regime in the sense that the magnetic fluctuations exceed kinetic fluctuations from the outset. Thus the analogue of the kinematic regime for the MF $\alpha^{2}$ case would be a "magnematic" regime where the small scale velocity fluctuations are small.

Numerical experiments in which the system is forced in the induction equation with an electric field that drives small scale magnetic helicity (Brandenburg et al. 2002; Alexakis et al. 2006: Park \& Blackman (2012b) show that indeed the large scale field grows in response. Park \& Blackman (2012b) compared the MF theory to MF numerical experiments and show that the buildup of the kinetic helicity explains the end of magnematic regime and quenches the MF analogue of the $\alpha^{2}$ dynamo. Thus kinetic and current helicity reverse their roles between the KF and MF cases. The growth of the large scale field in the MF vs. KF cases has another important distinction: Because of magnetic helicity conservation, the large scale field grows with the same sign as that injected for the MF case. In the KF case, the injection of kinetic helicity does not change the net magnetic helicity and so the large and small scale magnetic helicities grow with opposite signs.

The ratio of magnetic to thermal energy $\beta_{p} \geq 1$ at all times in the simulations of Park \& Blackman (2012b) and so the simulations thus demonstrate the existence of a $\beta_{p}>1$ magnetic fluctuation driven dynamo. Although only the simplest MF LSDs have been considered by employing no shear or rotation and periodic boundaries, the basic concept of a LSD that is driven by small scale magnetic flucations rather than kinetic fluctuatons in $\beta_{p}>1$ environments may have 
important conceptual relevance to stellar and accretion disk dynamos. In those cases, it should be noted however that the Coriolis force, anisotropic turbulence, and buoyancy conspire to allow still other contributions to the EMF. (e.g. Brandenburg \& Subramanian 2005).

Large scale dynamos are now commonly seen in accretion-disk-motivated simulations (Brandenburg et a.. 2005, Davis et al. 2010, Gressel 2010..41G, Käpylä and Korpi, 2011; Guan \& Gammie 2011). The MRI operating in these simulations produces turbulence that drives an LSD and the magnetic fluctations typically exceed the kinetic fluctuations. Agreement between LSD theory and simulation indeed seems to require consideration that the driver of the LSD growth is not the kinetic helicity term but may be the current helicity term (Gressel 2010). This would correspond to a $\beta_{p}>1$ magnetic fluctuation (or current helicity) driven dynamo. Note that the total flow kinetic energy also still dominates the magnetic energy because the Keplerian shear contains the dominant energy in the system. Moreover, in any thin disk, the helical fraction of the large scale field is expected to be subdominant in magnitude but still essential for diagnosing the LSD mechanism.

Note that in the $\beta_{p}<<1$ limit, MF LSDs have long been studied in the laboratory context (e.g. Bhattacharjee \& Hameiri 1986, Ji \& Prager 2002). The direct analogy to these low $\beta$ MF LSDs may occur in astrophysical coronae. We come back to this point later.

\subsection{Magnetic Flux Driven Dynamos}

An emerging frontier in 21st century astrophysical dynamo theory is the recognition of the importance of open boundaries because the electromotive force can be sustained exclusively by boundary terms. These boundary terms can continue to drive the EMF independent of the aforementioned quenching that arises because of competition between kinetic and current helicities.

To introduce the basic point consider the steady state limit of Consider now the limit of (5) and (6) in which the time evolution and resistive terms are ignored, but the divergence terms are kept. We then have respectively,

$$
0=2\langle\overline{\mathcal{E}} \cdot \overline{\mathbf{B}}\rangle-\nabla \cdot\langle\overline{\Phi \mathbf{B}}+\overline{\mathbf{E}} \times \overline{\mathbf{A}}\rangle
$$

and

$$
0=-2\langle\overline{\mathcal{E}} \cdot \overline{\mathbf{B}}\rangle-\nabla \cdot\langle\phi \mathbf{b}+\mathbf{e} \times \mathbf{a}\rangle
$$

Combining these two equations reveals that the fluxes of large and small scale helicity through the system boundary are equal and opposite in a steady state. In principle, the flux of small scale helicity can sustain an $\overline{\mathbf{B}}$-aligned EMF, $\overline{\mathcal{E}}_{\|}$, that grows the large scale field of oppositely signed helicity provided that there is a source of energy for the amplified magnetic field.

For a fixed amount of magnetic helicity, the energy in a magnetic structure is minimized when this helicity evolves to as large a scale as possible. Thus if the rate of helicity fluxes of small and 
large scales are equal, more small scale energy and current helicity would be leaving per unit time than that associated with the large scale field. If, for example, there is a source of EMF within the rotator from kinetic helicity, then a flux of small scale magnetic helicity can help alleviate the backreaction from the current helicity and allow the dynamo or cycle period to avoid quenching (e.g. Blackman \& Brandenburg 2003; Käpylä et al. 2010).

In general, both the time derivatives and the flux terms in equation (5) and (6) should be included dynamically. In the context of the Galaxy, Shukurov et al. (2006) solved the mean field induction equation for $\overline{\mathbf{B}}$ assuming that $\overline{\mathcal{E}}_{\|}$satisfies $\partial_{t} \overline{\mathcal{E}}_{\|}=0$, As discussed above, $\overline{\mathcal{E}}_{\|}$, involves the difference between the kinetic and current helicities which can be related to small scale magnetic helicity in the Coulomb gauge. (Shukurov et al. (2006) formally use a gauge invariant helicity density, derived by Subramanian \& Brandenburg (2006) to replace the use of the magnetic helicity density but the key role of the boundary terms is conceptually independent of this.), Shukurov et al. (2006) solve the induction equation for $\overline{\mathbf{B}}$, (which depends on $\overline{\mathcal{E}}_{\|}$and thus $\langle\mathbf{a} \cdot \mathbf{b}\rangle$ ) and Eq. (6) for $\langle\mathbf{a} \cdot \mathbf{b}\rangle$. They replace the divergence term in (6) with one of the form $\propto \nabla \cdot(\langle\mathbf{a} \cdot \mathbf{b}\rangle \overline{\mathbf{V}})$, where $\overline{\mathbf{V}}=\left(0,0, \bar{V}_{z}\right)$ is the mean velocity advecting the small scale helicity out of the volume. This mean velocity also appears in the induction equation for $\overline{\mathbf{B}}$, highlighting that the loss terms in the small scale helicity equation also imply advective loss of mean field. This approach exemplifies the general concept (Blackman \& Field 2000ab; Blackman 2003) that a flow of small scale helicity toward the boundary may help to alleviate the backreaction on the small scale magnetic helicity

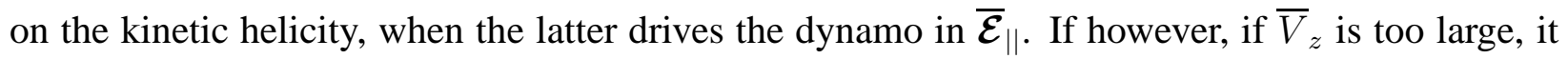
may carry away too much of the desired mean field which the dynamo is trying to grow in the first place.

A different time dependent dynamo that includes boundary terms, maintains the time dependence in (5), but implicitly assumes that equation (6) reaches a steady-state has also been studied (Vishniac \& Cho 2001; (See also Brandenburg \& Subramanian 2005 for further review). This explicitly incorporates the role of shear into the helicity flux and has been argued to be important in explaining angular momentum transport in accretion disks (Vishniac 2009,) Indeed, the role of some kind of shear dependent helicity flux may alleviate what appears to be the asymptotic vanishing of MRI turbulent stresses in unstratified shearing boxes at large magnetic Reynolds number (Vishniac 2009; Käpylä and Korpi 2011) and has been shown to alleviate catastrophic quenching in convection driven dynamos as well (Käpylä et al. 2010).

A subtle aspect of the helicity density equations (5) and (6), and thus for computing dynamos with open boundaries and helicity fluxes, is the issue of gauge invariance. In the absence of boundary flux terms, all terms are manifestly gauge invariant. In the present of flux terms, the gauge invariance is broken (e.g. Berger \& Field 1986). Subramanian \& Brandenburg (2006) carefully construct a generalized local helicity density that reduces to the above equations in the absence of flux terms and has the same form in the presence of flux terms but with a redefinition of $H_{1}^{M}$, 
$H_{2}^{M}$ and the flux terms that avoids the use of the vector potential. The lowest order terms in their definition are similar to what is obtained from the Coulomb gauge but the corrections make each individual terms in the equation gauge invariant with a local physical meaning. That important construction puts the physical discussion of helicity density evolution equations here and elsewhere on firm ground, particularly if one seeks to measure a local flux density as a physical quantity. However, if one uses the helicity evolution equations as intermediary equations in a particular gauge and can convert back to the magnetic field, the resulting magnetic field will always be gauge independent.

Numerical simulations may be easier to carry out in particular gauges and the specific choices of boundaries may be troublesome for specific gauges. In this context, as emphasized by Hubbard \& Brandenburg (2011), the specific Vishniac-Cho (VC) flux is typically computed in the Coulomb gauge. Hubbard \& Brandenburg (2011) have argued that the Coulomb gauge is problematic numerically for periodic shear flows. Appealing to the fact that flux divergences can be gauge invarian even when fluxes are not, and working in a numerically appealing shear-periodic gauge they find that for the unstratified periodic box conditions previously though to incur a VIshniac-Cho flux, the vertical flux vanishes even though the divergence of the total flux does not. They argue that the VC flux is a horizontal flux and not a vertical one, and therefore would not be useful to large scale dynamos as a small scale helicity release valve. It may be that the enhanced turbulent diffusion of small scale helicity (e.g. Blackman 2003; Hubbard \& Brandenburg 2010) plays a more prominent role.

A further subtlety is that Hubbard and Brandenburg (2012) have pointed out that shearperiodic boundaries lead to helicity fluxes that that flip sign at what should otherwise be shear periodic boundaries. (This is a bit troubling in the analogous way that vertically stratified periodic boxes have to set gravity to zero at the top and bottom boundaries to be sensible). In a horizonally averaged analysis, they purport to avoid dealing with these fluxes by working with a set of equations that avoids solving the time evolution of the small scale magnetic helicity. In this approach, the flux terms hidden, working "behind the scenes" to prevent the dynamo from quenching prematurely. However, with K. Subramanian (K. Subramanian 2012, personal comm.) we have found that there seem to be flux terms that are dropped form their alternative set of equations both in their horizontally averaged case and for more general extensions which do not seem to be easily argued to vanish so more work is needed to assess whether helicity fluxes work can really be thought to work "behind the scenes" or need to be explicitly calculate. The issue is of practical importance, while the alpha-squared dynamo can grow sizeable mean field and saturate at that value even without helicity fluxes, the question of whether an $\alpha-\Omega$ dynamo requires helicity fluxes to reach a steady state with a sizeable mean field before that field rapidly decays is directly related to this issue. (e.g. compare Sur et al. 2007; to Hubbard and Brandenburg 2012). There is more to be done to quantify helicity flux driven LSDs and corroborate theory and numerical simulation. 
Magnetic helicity fluxes into astrophysical coronae, and the subsequent evolution of such structures are directly analogous to the evolution magnetically dominated laboratory plasma sub-

ject to the injection of magnetic helicity as in a Spheromaks (e.g. (Bellan 2000), Hsu \& Bellan 2002) or reversed field pinches (Ji \& Prager 2002). Such relaxation process have been studied as helicity flux driven dynamos in magnetically dominated plasmas (e.g. Bhattacharjee \& Hameiri 1986).

The experiment of Hsu \& Bellan 2002 provides an analogy to helical loops of flux rising into an astrophysical corona from its rotator below. The loops coalesce at the symmetry axis and form a magnetic tower. For large enough helicity injection, the tower can break off a Spheromak blob from the kink instability. An astrophysical corona can be modeled as an aggregate of magnetic loops subject to reconfiguration, reconnection, and relaxation (Blackman \& Field 04; Uzdensky \& Goodman 2008). The helicity flux to the corona in astrophysical rotators acts as a seed for this subsequent magnetic relaxation (or MDHD) Field lines that open up global may be those which enable jets.

\section{Accretion Theory and the Connection to LSDs}

\subsection{Standard accretion theory is a mean field theory}

Gaseous accretion discs around astrophysical rotators have long been studied as a source of luminosity (see Treves et al. 1989; Frank et al. 2002). In astronomy, a practical semi-analytic formalism to be incorporated into emission models for comparison with observations is a desired goal. Practical accretion models in standard texts (e.g. Frank et al. 2002) based on SS73 employ the Navier-Stokes equation and replaces the microphysical viscosity with a turbulent viscosity without explaining the theoretical complexity and assumptions underpinning this bold procedure. The replacement of the microphysical viscosity by a turbulent viscosity is itself a turbulent closure and warrants a formal derivation analogous to that of the MFD theory coefficients. Closures approximate an otherwise infinite set of nonlinear turbulence equations by an approximation that facilitates a finite set.

That the SS73 model is both axisymmetric AND involves a turbulent viscosity implies that the theory is a mean field theory because local axisymmetry never applies on the scale of turbulent fluctuations. That accretion disc theory is a mean field theory is addressed in a subset of theoretically oriented literature (e.g. Balbus et al. 1994; Blackman 1997; Balbus \& Hawley 1998; Ogilvie 2003 Pessah et al. 2006; Hubbard \& Blackman 2009). MFD theory has traditionally focused on the magnetic induction equation as the "primary equation" of the theory and accretion disc models focus on the mass conservation and angular momentum transport equations as the "primary equations" of the theory with the coupling to the magnetic field often ignored or "swept" into " $\alpha_{s s}$ ". At present, a formal identification of the minimal set of transport coefficients that best predicts the 
angular momentum transport seen in simulations and can be used for practical spectral modeling is lacking.

MFD theory is more progressed than mean field accretion theory because there is a semianalytic MFD theory that agrees with a set of nonlinear simulations, as discussed earlier. Recall from section 2 that MFD theory has presently 2 tensor coefficients in common use (the $\alpha_{i j}$ and $\beta_{i j k}$ coefficients which are used even in semi-analytic dynamo models to match observed field structures in stars or in galaxies. Dynamical equations that incorporate magnetic helicity evolution and employ "minimal $\tau$ " closure (e.g. Blackman \& Field 2002, see also Snellman et al. 2009 applied to shear flows) capture MFD saturation. In accretion theory however, there are few models that use anything other than the single diffusion coefficient of the SS73 formalism. The minimum properties that turbulence must have to transport angular momentum outward are more subtle than what purely isotropic turbulence provides, and it is likely that more than one transport coefficient is needed (Pessah et al. 2006; Hubbard \& Blackman 2009). Progress toward capturing the saturated stress of the magneto-rotational instability (MRI) as a mean field model using a closure similar to the "minimal $\tau$ " approximation and going beyond the $\alpha_{s s}$ disc model may be slowly emerging (Ogilvie 2003; Pessah et al. 2006).

Although the magneto-rotational instability (MRI) has emerged as a leading candidate for local angular momentum transport in accretion discs (e.g. Balbus \& Hawley 1998; Balbus 2003), we do not yet have quantitative scalings for the measured transport coefficients that can be used in practical disk emission models. Simulations have not converged to the extent that robust scalings of transport coefficients can be extracted (Hawley et al. 2011). Perhaps even more substantially, since most MRI "accretion disk" simulations have neither disks (they are cartesian boxes) nor accretion (there is no torque) even if we were able to obtain converged stresses, we still would be deficient in determining torques. The latter really requires more global simulations. In particular, there is a need to determine the relative fraction of vertical torque that transports angular momentum vertically compared to radially, and the subsequent fraction of that vertical transport that divides into corona vs. jet.

Most first generation MRI simulations (except Brandenburg et al.1995) also did not use explicit viscosity or magnetic diffusivity. Indeed more recent work does show that for unstratified simulations there is a magnetic Prandtl number dependence (Fromang et al. 2007; Lesur and Longaretti 2007). The dimensionless transport parameter $\alpha_{s s}$ depends strongly on the box size and the strength of the initially imposed weak mean field strength for unstratified boxes with imposed mean fields (Pessah et al. 2007), though though this dependence is reduced for stratified boxes (Davis et al. 2010; Shi et al. 2009). Interestingly, $\alpha_{s s}$ varies $\sim 4$ orders between simulations, but $\alpha_{s s} \beta_{p}$, where $\beta_{p}$ is the ratio of thermal to magnetic pressure, is nearly a constant (Blackman et al. 2008). This is maintained in global simulations as well (e.g. Romanova et al. 2011)

The mean field approach of Pessah et al. (2006) hints at what minimal set of coefficients 
might be required in a more robust but still practical model of MRI mediated accretion discs. The approach better matches the stress dependence on rotation profile than the $\alpha_{s s}$ of SS73 in numerical simulations of unstratified boxes with an initial mean field (Pessah et al. 2008). .

\subsection{Role of Large Scale Fields: Clues from MRI Simulations}

Due to computational limitations, much of the early computational study of the promising MRI has focused on the local manifestation of the instability as a source of local turbulence that can transport angular momentum radially outward (e.g. Balbus \& Hawley 1998, Balbus 2003) Understanding the local physics is important but even classic paradigms of accretion discs involve large scale magnetic loops or global fields (Lynden-Bell 1969; FIeld \& Rogers 1993; Tout \& Pringle 1992; Johansen and Levin 2008) or outflows (Blandford \& Payne 1982; Königl 1989, Tager \& Pellat 1999; Colgate et al. 2001; Caunt \& Tagger 2001 Königl et al. 2010) as primary transport agents. There have also been a few efforts to incorporate the dynamics of vertical transport and its effect on the radiation spectra in semi-analytic models of accretion disks (Kuncic \& Bicknell 2004,2007; Dobbie et al. 2009).

Mesoscale shearing box simulations (Davis et al. 2010; Guan \& Gammie 2011; SImon et al. 2012). and global accretion disk simulations (Penna et al. 2010, Beckwith et al. 2011, Sorathia et al. 2010, Tchekhovskoy et al. 2011, Romanova et al. 2011) are becoming more numerous but convergence is still an issue (Hawley et al. 2011; Bodo et al. 2011) and compromises still have to be made. For example, simulations may include stratification but not explicit resistivity and viscosity (or vice versa). The choice of periodic vs. open vertical boundaries simulations is another distinction with practical implications. For global disk simulations, the computational time limitations typically require choosing an initial seed magnetic field that is relatively large. Assessment of which results from simulations depend on initial conditions and boundary conditions needs to be further investigated. The torques rather than the stresses are the most relevant quantities to determine for angular momentum transport.

Stratified local or meso-scale simulations do show less dependence on resolution and box size than unstratified solutions (Pessah et al. 2007; Bodo et al. 2008; Davis et al. 2010) though are seemingly still not absolutely converged (Guan \& Gammie 2011; Hawley et al. 2011) Since real systems are stratified, the weaker dependence of the saturated state on box properties is step forward, but with stratification comes magnetic buoyancy and coronae. Large scale structures have the longest turbulent diffusion times and are most likely to survive the buoyant rise (Blackman $\&$ Pessah 2009). Thus if stratification is important then understanding what radial and vertical scales are large enough to fully capture the dominant magnetic stress remains a question for global simulations.

Global mean toroidal magnetic fields that last many orbit times and exhibit sign reversals are 
now seen routinely in local shearing box simulations (Brandenburg et al. 1995, Lesur \& Ogilvie 2008, Davis et al. 2010; Guan et al. 2011; Oishi and MacLow 2011; Simon et al. 2012) and in global simulations (Penna et al. 2010 ,Beckwith et al. 2011, Sorathia et al. 2010, Tchekhovskoy et al. 2011, Romanova et al. 2011). The cycles are evident in in stratified simulations with and without periodic vertical boundaries (Oishi and MacLow 2011), and even in unstratified simulations with periodic vertical boundaries (Lesur \& Ogilvie 2008). The patterns indicate a LSD operating contemporaneously with the small scale dynamo that produces magnetic fluctuations. Understanding the extent to which the large scale fields transport angular momentum vertically and non-locally is a desired pursuit. In all of these cases however, there is at minimum a Coriolis force which presents a pseudo-scalar $\Omega \cdot \hat{\mathbf{z}}$ and anisotropy which can source an EMF, and in turn , sources magnetic helicity growth. (See also Rüdiger and Pipin 2000; Rekowski et al. 2000; Brandenburg and Subramanian 2005).

It is important to note several principles in analyzing shearing box simulations for LSD action and the magnetic helicity. FIrst, when there is a mid-plane, any pseudoscalars or reflection asymmetric quantities reverse sign. When any helicities are computed by averaging over the entire box one expects that such quantities will vanish (regardless of the boundary conditions). In addition, the helicities may have opposite signs on different scales even within a given hemisphere. Thus one should separately compute helicity spectra in each hemisphere to analyze their role. This is in addition to evaluating any boundary terms. In this context note that Oishi \& MacLow (2011) found that the stratified periodic and stratified open boundary simulations are similar with respect to LSD action which is an important result. However, since their analysis of the possible role of magnetic helicity did not involve the separate analysis of helicity in each hemisphere we cannot yet rule out the role magnetic helicity dynamics as being fundamental to the LSD action in the simulations they compare. Even with a closed box, local helicity fluxes may be important. A further point is the helical fraction of the magnetic field is not a measure of the importance of magnetic helicity in that we expect shearing boxes to be have predominantly non-helical fields due to shear. The needed LSD process by which some toroidal field is converted to poloidal field and sustains the field against decay may still depend fundamentally on a dynamics that tracking magnetic helicity evolution may help explain.

\section{Combining MFD and Accretion Theory}

Here I outline the basic ingredients of a "strawman" paradigm for sufficiently ionized, nonself-gravitiating accretion engines that combine the MRI and LSDs. There are two flavors of scenarios: (1) those led by an EMF supplied by pseudocalars fluctuations and aided by helicity fluxes or (2) those fully dominated by helicity fluxes with pseducalar contributions to the EMF 
small. Fluxes are the common ingredient to both paradigms.

For a disk that becomes unstable to MRI driven turbulence, stratification and rotation in a real system then imply a source of reflection asymmetry. Because the MRI produces magnetic fluctuations that dominate velocity fluctuations one pseudoscalar of relevance is the current helicity. Provided there is sufficient current helicity from these magnetic fluctuations, a current helicity driven LSD can result. Such an LSD that is driven by current helicity fluctuations (rather than kinetic helicity) is conceptually consistent with a generalization the $\beta_{p}>1$ magnetic fluctuation driven dynamo discussed in section 3.1 to include shear and may be a plausible model for LSDs observed in some MRI simulations (Gressel 2010).

The combination of the small scale magnetic fluctuations driven by the MRI and the current helicity driven LSD will produce a spectrum of magnetic energy and a spectrum of magnetic stress. Magnetic structures large enough to buoyantly rise 1-2 density scale heights before being shredded by turbulent diffusion are the structures that form the corona (Blackman \& Pessah 2009). The vertical angular momentum transport likely results only from the structures that satisfy the buoyancy condition so there would be a 1 to 1 correspondence between the fraction of total magnetic stress associated with these structures and the ratio of vertical to total stress. (Derivation of the correlation length of magnetic fields from MRI simulations is a useful goal in this context such as in Simon et al. 2012). The local radial stress would be due to magnetic structures who scale is below the critical scale for buoyancy. It is desirable to extract the ratio of vertical to total stress from simulations and compare to the corresponding properties of the magnetic stress spectra for structures above and below the critical scale for buoyancy. It would also be of interest to determine the non-local radial stress from large scale loops anchored at different radii. The torques associated with the vertical and non-local stresses could be comparable to the local radial torque even if the stress ratio is small since vertical gradients can be larger than radial gradients.

Magnetic structures large enough scale to survive the buoyant rise to the corona also have magnetic helicity and even likely have some amount of both positive and negative sign by analogy to the sun (Blackman \& Brandenburg 2003; Brandenburg et al. 2011 ). The flow of magnetic helicity to the corona from the disk is capture by a flux term that can sustain $\overline{\mathcal{E}}_{\|}$as discussed in section 3.2. When fields reach the corona, they can further relax via dynamical magnetic relaxation (MDHD) in what would be a $\beta_{p}<1$ environment. There will be some competition between relaxation to large scales and dissipation when loops of opposite sign reconnect, and ultimately some coronal spectrum will result (see also Uzdensky \& Goodman 2008, who did not however invoke magnetic helicity conservation). The largest scale fields will mediate the jets and the coronal loops will be agents of hard X-ray emission (at least in AGN disks) upon reconnection (e.g. Field $\&$ Rogers 1993). Whether the helicity fluxes that are accompanied by a flux of magnetic energy help sustain the pseudocalar driven EMF as in e.g. Sur et al. 2007 or by themselves sustain the LSD as in e.g. Vishnical (2009) is unclear at present. 
As just described, the transport of angular momentum involves both radial and vertical components. The local radial component would leads to thermal disk emission and the vertical and (and some non-local radial) would contribute to jets and coronae. Both large scale fields and the small scale fields are then important . A generalized mean field accretion disk theory must therefore start with equations for the mean and fluctuating components of the magnetic field and the velocity and employ a suitable closure to reduce the set of mean field equations to closed form, leaving a finite set of transport coefficients. In this way, corona, jet, and disk emission can all be coupled into a single theory. I think that finding such a theory is tractable. At first one may have to compromise aspects of a dynamical theory for parameterization informed by simulations, but the ultimate goal would be to determine the underlying principles behind each transport coefficient. One can envision both 1-D (vertically integrated, azimuthally averaged) and 2-D (only azimuthally averaged) theories. Note that issues such as the competition between diffusion of global scale magnetic fields in accretion discs, advection of flux, and amplification of flux are all part of the same question: how do mean magnetic fields evolve in accretion discs when the appropriate minimal set of mean field transport coefficients are obtained for both the coupled LSD and accretion dynamics? Ultimately, mean field accretion disc theory and LSD theory are coupled.

Some aspects of the coupled evolution of mean velocity field in accretion flows and mean magnetic fields in dynamo theory have been studied in semi-analytic models. However, principles of 21 st century LSD theory have yet to be included in this coupling. Campbell \& Caunt (1999) combined 20th century MFD theory with the SS73 accretion theory closure to include local and large scale stresses and Campbell (2003) studied the vertical and radial accretion disk structure coupled to an LSD supplied magnetic field and magnetized wind. Kuncic \& Bicknell $(2004,2007)$ have derived a mean field MHD accretion theory that includes radial and vertical transport and have calculated the effect on the thermal disk spectrum and can accommodate the limit in which all of the transport is vertical (pure Poynting flux accretion solution). They have not included LSD theory or a detailed coronal + jet spectal model. Quataert \& Narayan (1999) included spectra from both disks and winds for advection dominate flows but without including the MHD dynamics or an LSD theory. The SS73 type viscosity (via MRI) has also been used as a closure for the turbulence from which semi analytic MFDT is then derived to produce large scale fields in discs to power outflows (e.g. Tan \& Blackman 2004). 20th century MFD theory has also been applied to MRI stratified simulations to explain observed cycle periods (Brandenburg et al. 1995; Brandenburg \& Donner 1997; also Lesur \& Ogilvie 2008; Davis et al. 2010; Guan \& Gammie 2011).

There is much more semi-anlaytic work to be done. In fact, as simulations become more and more sophisticated, the opportunities for analytic and semi-analytic work increases. 


\section{Conclusions}

For any turbulent MHD system in which there is both turbulence as well as ordered large scale patterns, there exists an averaging and appropriate closure that dynamically captures essential physics and key principles in a useful framework. It may not be easy to find this closure but the question should not be "is mean field theory is correct?" but "do we have the correct mean field theory?" Progress in large scale dynamo theory is farther along that accretion theory in this regard; mean field dynamo theories now exist that make correct predictions of the nonlinear saturation of simple large scale dynamos but we do not yet have a theory for accretion disks that has either identified the complete set of transport coefficients, or predicts their time evolution. Noteworthy progress this goal is the model described for periodic box MRi simulations in Pessah et al. (2008). However, the ultimate desired theory needs to couple mean field accretion theory with mean field dynamo theory in such a way that the vertical and large scale angular momentum transport, evidenced by coronae and jets, are captured along with small scale local transport within the disk.

It is plausible that the MRI, operating in a stratified rotator with open boundaries, generates a magnetic spectrum which has both: (1) small scale MHD turbulence with magnetic structures below the scale for which buoyancy beats turbulent shredding. These structures transport angular momentum radially and account for dissipation within the disk; (2) larger scale magnetic structures for which buoyancy beats turbulent diffusion and that transport angular momentum to the coronae. Present simulations suggest that large scale helical dynamos operate within accretion disks, driven in part by current helicity fluctuations produced by the MRI. The flux of magnetic helicity from disk to corona can help sustain the large scale dynamo within disk and feed the disk corona with

magnetic fields. A fraction of the magnetic fields that make it to the corona relax to open up and mediate jets. I think that it will be possible to develop a mean field theory with a finite set of useful transport coefficients that can accommodate all of these ingredients and in fact still be practical for use by observational modelers.

Thanks to K. Subramanian, O Gressel, F. Naumann, and R. Penna, J. Oishi, and K. Park for discussions. I acknowledge NSF grants AST-0406799, AST-0406823, and NASA grant ATP04 -0000-0016 and the LLE at UR. Thanks to the organizers of the "Turbulent Mixing and Beyond" meeting in 2011 in Trieste, Italy for a stimulating meeting.

\section{REFERENCES}

Alexakis, A., Mininni, P. D., \& Pouquet, A. 2006, ApJ, 640, 335

Balbus, S. A., \& Hawley, J. F. 1998, Reviews of Modern Physics, 70, 1 
Balbus, S. A. 2003, ARA\&A, 41, 555

Balbus, S. A., Gammie, C. F., \& Hawley, J. F. 1994, MNRAS, 271, 197

Beck, R., Brandenburg, A., Moss, D., Shukurov, A., \& Sokoloff, D. 1996, ARA\&A, 34, 155

Beckwith, K., Armitage, P. J., \& Simon, J. B. 2011, MNRAS, 416, 361

Bellan P.M., 2000, Spheromaks, (Imperial College Press, London)

Berger, M. A., \& Field, G. B. 1984, Journal of Fluid Mechanics, 147, 133

Bhattacharjee, A., \& Hameiri, E. 1986, Physical Review Letters, 57, 206

Blackman E. G., Field G. B., 2000a, ApJ, 534, 984

Blackman, E. G., \& Field, G. B. 2000b, MNRAS, 318, 724

Blackman, E. G., \& Field, G. B. 2002, Physical Review Letters, 89, 265007

Blackman, E. G., \& Brandenburg, A. 2003, ApJ, 584, L99

Blackman, E. G. 2003, Turbulence and Magnetic Fields in Astrophysics, 614, 432

Blackman, E. G. 2004, Plasma Physics and Controlled Fusion, 46, 423

Blackman, E. G., \& Field, G. B. 2004, Physics of Plasmas, 11, 3264

Blackman, E. G. 2007, New Journal of Physics, 9, 309

Blackman, E. G., Penna, R. F., \& Varnière, P. 2008, New Astronomy, 13, 244

Blackman, E. G., \& Pessah, M. E. 2009, arXiv:0907.2068, ApJ, 704 L113

Blackman, E. G. 2010, Astronomische Nachrichten, 331, 101

Blandford, R. D., \& Payne, D. G. 1982, MNRAS, 199, 883

Bodo, G., Mignone, A., Cattaneo, F., Rossi, P., \& Ferrari, A. 2008, A\&A, 487, 1

Bodo, G., Cattaneo, F., Ferrari, A., Mignone, A., \& Rossi, P. 2011, ApJ, 739, 82

Brandenburg, A., Nordlund, A., Stein, R. F., \& Torkelsson, U. 1995, ApJ, 446, 741

Brandenburg, A., \& Donner, K. J. 1997, MNRAS, 288, L29 
Brandenburg, A. 1998, in "Theory of Black Hole Accretion Disks," edited by M.A. Abramowicz, G.Bjornsson, and J. E. Pringle. Cambridge University Press, 1998., p.61

Brandenburg, A. 2001, ApJ, 550, 824

Brandenburg, A., Dobler, W., \& Subramanian, K. 2002, Astronomische Nachrichten, 323, 99

Brandenburg, A., \& Subramanian, K. 2005, Phys. Rep., 417, 1

Brandenburg, A., Rädler, K.-H., Rheinhardt, M., Käpylä, P. J. 2008, ApJ, 676, 740

Brandenburg A., Subramanian K., Balogh A., Goldstein M. L., 2011, ApJ, 734, 9

Campbell, C. G., \& Caunt, S. E. 1999, MNRAS, 306, 122

Campbell, C. G. 2003, MNRAS, 345, 123

Caunt, S. E., \& Tagger, M. 2001, A\&A, 367, 1095

Cattaneo, F. 1994, ApJ, 434, 200

Colgate, S. A., Li, H., \& Pariev, V. 2001, Physics of Plasmas, 8, 2425

Davis, S. W., Stone, J. M., \& Pessah, M. E. 2010, ApJ, 713, 52

Dobbie, P. B., Kuncic, Z., Bicknell, G. V., \& Salmeron, R. 2009, arXiv:0903.3274

Field, G. B., \& Blackman, E. G. 2002, ApJ, 572, 685

Field, G. B., \& Rogers, R. D. 1993, ApJ, 403, 94

Frank, J., King, A., \& Raine, D. J. 2002, Accretion Power in Astrophysics, by Juhan Frank and Andrew King and Derek Raine, pp. 398. ISBN 0521620538. Cambridge, UK: Cambridge University Press, February 2002.,

Fromang, S., Papaloizou, J., Lesur, G., \& Heinemann, T. 2007, A\&A, 476, 1123

Gressel, O. 2010, MNRAS, 405, 41

Guan, X., \& Gammie, C. F. 2011, ApJ, 728, 130

Hawley, J. F., Guan, X., \& Krolik, J. H. 2011, ApJ, 738, 84

Heinemann, T., McWilliams, J. C., \& Schekochihin, A. A. 2011, Physical Review Letters, 107, 255004 
Hubbard, A., \& Blackman, E. G. 2008, MNRAS, 390, 331

Hubbard, A., \& Blackman, E. G. 2009, MNRAS, 398, 931

Hubbard, A., \& Brandenburg, A. 2010, Geophysical and Astrophysical Fluid Dynamics, 104, 577

Hubbard, A., \& Brandenburg, A. 2011, ApJ, 727, 11

Hubbard A., Brandenburg A., 2012, ApJ, 748, 51

Ji H., \& Prager S.C., 2002, Magnetohydrodynamics 38, 191

Johansen, A., \& Levin, Y. 2008, A\&A, 490, 501

Käpylä, P. J., Korpi, M. J., \& Brandenburg, A. 2008, A\&A, 491, 353

Käpylä, P. J., Korpi, M. J., \& Brandenburg, A. 2010, A\&A, 518, A22

Käpylä, P. J., \& Korpi, M. J. 2011, MNRAS, 413, 901

Kleeorin, N. I. \& Ruzmaikin, A. A., 1982 Magnetohydrodynamics 18, 116

Krause F. and RŁdler K. H. Mean Field Magnetohydrodynamics and Dynamo Theory 1980, Pergamon Press.

Kolekar S., Subramanian K., Sridhar S., 2012, arXiv, arXiv:1204.2714

Konigl, A. 1989, ApJ, 342, 208

Königl, A., Salmeron, R., \& Wardle, M. 2010, MNRAS, 401, 479

Kuncic, Z., \& Bicknell, G. V. 2004, ApJ, 616, 669

Kuncic, Z., \& Bicknell, G. V. 2007, Ap\&SS, 311, 127

Lesur, G., \& Ogilvie, G. I. 2008, A\&A, 488, 451

Lesur, G., \& Longaretti, P. Y. 2007, MNRAS, 378, 1471

Lovelace, R. V. E., Rothstein, D. M., \& Bisnovatyi-Kogan, G. S. 2009, ApJ, 701, 885

Lynden-Bell, D. 1969, Nature, 223, 690

Moffatt, H. K. 1978, Cambridge, England, Cambridge University Press, 1978. 353 p.,

Ogilvie, G. I. 2003, MNRAS, 340, 969 
Oishi, J. S., \& Mac Low, M.-M. 2011, ApJ, 740, 18

Park, K., \& Blackman, E. G. 2012a, MNRAS, 419, 913

Park, K., \& Blackman, E. G. 2012b, arXiv:1201.0800

Parker, E. N. 1979, Oxford, Clarendon Press; New York, Oxford University Press, 1979, 858p, Cosmical magnetic fields: Their origin and their activity

Penna, R. F., McKinney, J. C., Narayan, R., et al. 2010, MNRAS, 408, 752

Pessah, M. E., Chan, C.-K., \& Psaltis, D. 2006, Physical Review Letters, 97, 221103

Pessah, M. E., Chan, C.-k., \& Psaltis, D. 2007, ApJ, 668, L51

Pessah, M. E., Chan, C.-K., \& Psaltis, D. 2008, MNRAS, 383, 683

Pessah, M. E., \& Goodman, J. 2009, ApJ, 698, L72

Pietarila-Graham, J., Blackman, E. G., Mininni, P. D., \& Pouquet, A., in press Phys Rev E, 2012.

Pouquet A., Frisch U., Léorat J., 1976, J. Fluid Mech., 77321

Quataert, E., \& Narayan, R. 1999, ApJ, 520, 298

Rogachevskii, I., \& Kleeorin, N. 2003, PRE, 68, 036301

Romanova, M. M., Ustyugova, G. V., Koldoba, A. V., \& Lovelace, R. V. E. 2011, arXiv:1111.3068

Rüdiger, G., \& Pipin, V. V. 2000, A\&A, 362, 756

Schrijver C.J. \& Zwaan C., 2000, Solar and Stellar Magnetic Activity, (Cambridge: Cambridge Univ. Press)

Shakura, N. I., \& Syunyaev, R. A. 1973, A\&A, 24, 337 (SS73)

Shi, J.-M., Krolik, J. H., \& Hirose, S. 2009, arXiv:0909.2003, submitted to ApJ.

Shukurov, A. 2002, Ap\&SS, 281, 285

Shukurov A., Sokoloff D., Subramanian K., Brandenburg A., 2006, A\&A, 448, L33

Simon J. B., Beckwith K., Armitage P. J., 2012, MNRAS, 422, 2685

Snellman, J. E., Käpylä, P. J., Korpi, M. J., \& Liljeström, A. J. 2009, arXiv:0906.1200

Sorathia, K. A., Reynolds, C. S., \& Armitage, P. J. 2010, ApJ, 712, 1241 
Subramanian, K., \& Brandenburg, A. 2006, ApJ, 648, L71

Sur, S., Shukurov, A., \& Subramanian, K. 2007, MNRAS, 377, 874

Sur, S., \& Subramanian, K. 2009, MNRAS, 392, L6

Tagger, M., \& Pellat, R. 1999, A\&A, 349, 1003

Tan, J. C., \& Blackman, E. G. 2004, ApJ, 603, 401

Tchekhovskoy, A., Narayan, R., \& McKinney, J. C. 2011, MNRAS, 418, L79

Taylor, J. B. 1986, Reviews of Modern Physics, 58, 741

Tout, C. A., \& Pringle, J. E. 1992, MNRAS, 259, 604

Treves, A., Maraschi, L., \& Abramowicz, M. 1989, Accretion: A Collection of Influential Papers Advanced Series in Astrophysics and Cosmology, 5, (World Scientific: Singapore)

Uzdensky, D. A., \& Goodman, J. 2008, ApJ, 682, 608

Vallée, J. P. 2004, New Astronomy Review, 48, 763

Vishniac, E. T., \& Brandenburg, A. 1997, ApJ, 475, 263

Vishniac, E. T., \& Cho, J. 2001, ApJ, 550, 752

Vishniac, E. T. 2009, ApJ, 696, 1021

Wang, Y.-M., \& Sheeley, N. R., Jr. 2003, ApJ, 599, 1404

Yousef, T. A. et al., 2008, Phys Rev. Lett., 100, 184501 